\title{
Isotropic and anisotropic description of superconducting state in $\mathrm{CaC}_{6}$ compound
}

\author{
R. Szczȩśniak ${ }^{1}$, E.A. Drzazga ${ }^{1}$, and D. Szczȩśniak ${ }^{2, a}$ \\ 1 Institute of Physics, Częstochowa University of Technology, Al. Armii Krajowej 19, 42-200 Częstochowa, Poland \\ 2 Institute of Physics, Jan Długosz University in Czȩstochowa, Al. Armii Krajowej 13/15, 42-200 Czȩstochowa, Poland
}

Received 10 September 2014 / Received in final form 9 January 2015

Published online 18 February 2015

(C) The Author(s) 2015. This article is published with open access at Springerlink.com

\begin{abstract}
The thermodynamic parameters of the superconducting state in $\mathrm{CaC}_{6}$ have been determined in the framework of the isotropic and anisotropic Eliashberg formalism. The obtained results determine the anisotropy effect on the values of the thermodynamic functions. In particular, it has been found that the anisotropy of the electron-phonon coupling constant and the Coulomb pseudopotential significantly affects the order parameter and the wave function renormalization factor, which determines the electron effective mass. These results mean that anisotropy should be also visible in the total normalized density of states. In the case of the thermodynamic critical field, anisotropy lowers its value in the low-temperature area. On the other hand, it does not affect the specific heat jump at the critical temperature.
\end{abstract}

\section{Introduction}

The classical mechanism of the formation of the superconducting state is related to the interaction occurring between the electron gas and the crystal lattice vibrations (phonons) $[1,2]$.

In the case when the value of the electron-phonon coupling constant $(\lambda)$ is not higher than 0.3 , the thermodynamic properties of the superconducting phase can be successfully described by the BCS theory [3,4]. Let us notice that this theory is based on the Hamiltonian, which eliminates the phonon degrees of freedom and uses the mean field approximation.

For the physical systems, in which $\lambda>0.3$, the thermodynamics of the superconducting state can be relevantly dependent on the strong-coupling and the retardation effects. Such effects are included in the Eliashberg formalism, which is the natural generalization of the BCS theory [5].

In particular, the respective equations (Eliashberg equations) have been derived straight from the Hamiltonian, which models the linear interaction between the electron gas and the phonons, applying at the same time the method of the thermodynamic Green's functions $[1,2,6-8]$. The result is the non-linear system of equations for the order parameter and the wave function renormalization factor, which structure allows the quantitative description of the strong-coupling and the retardation effects.

\footnotetext{
a e-mail: d.szczesniak@ajd.czest.pl
}

The classical superconductors, in which the Cooper pairs condensate is induced by the electron-phonon interaction, are characterized by a low value of the critical temperature $\left(T_{C}\right)$ [7]. This fact strongly limits the possibility of their industrial application. Thus, for many years there have been constant attempts made to find the physical systems, in which the superconducting state would be characterized by the highest possible $T_{C}$ value.

In the case of the systems with the electron-phonon pairing mechanism, some kind of a breakthrough came in 2001, when the superconducting properties of magnesium diboride were discovered [9]. In particular, it was found that the high value of the critical temperature in $\mathrm{MgB}_{2}$ $\left(T_{C}=39.4 \mathrm{~K}\right)$ results from the existence of the two-band electronic structure ( $\sigma$ and $\pi$ band) [10]. It is worth mentioning that the anisotropy of the electronic properties of the considered system additionally results in significant deviations of the thermodynamic parameters from the predictions of the BCS theory [11].

The recently conducted numerical calculations suggest also that the strong electronic anisotropy can be responsible for the formation of the high-temperature superconducting state in molecular metallic hydrogen. For example, for the pressure at $414 \mathrm{GPa}$, the estimated value of the critical temperature is equal to $84 \mathrm{~K}$ [12-14]. In the present case, three effective bands have been distinguished. Thus, for the proper description of the thermodynamic parameters in hydrogen, the three-band Eliashberg formalism is required [15].

In the case of the standard pressure, the only known three-band electron-phonon coupled superconductor is 
$\mathrm{CaC}_{6}$ compound [16-19]. In particular, $\mathrm{CaC}_{6}$ represents the superconductor of the intermediate anisotropy.

We want to underline that at the moment $\mathrm{CaC}_{6}$ is the graphite intercalated compound with the highest critical temperature at the ambient pressure: $T_{C}=11.5 \mathrm{~K}[16]$. It should be also noted that the critical temperature of $\mathrm{CaC}_{6}$ can be further modified with increasing pressure. Many theoretical and experimental investigations proved that $T_{C}$ in $\mathrm{CaC}_{6}$ exhibits an interesting increasing-decreasingincreasing behavior under pressure $[18,20-24]$. In particular, the critical temperature increase linearly to the value of $15.1 \mathrm{~K}$, when the pressure increases to $7.5 \mathrm{GPa}$. Next, at $8 \mathrm{GPa}$, the transition temperature drops and levels off at $5 \mathrm{~K}$ above $10 \mathrm{GPa}[22]$, due to the structural phase transition. Between 18 and $32 \mathrm{GPa}$ no superconducting transition above $2 \mathrm{~K}$ is observed [23].

Recently however, $\mathrm{Li}$ et al. have reported the reemergence of the superconductivity in the heavily compressed $\mathrm{CaC}_{6}(p \simeq 39 \mathrm{GPa})$, applying pressure via the theoretical calculations [24]. In particular, the authors have stated that the predicted phase III (space group Pmmn) with the formation of the carbon nanofoam is found to be stable at a wide pressure range with the critical temperature up to $14.7 \mathrm{~K}$ at $78 \mathrm{GPa}$.

The proof that the pairing mechanism in $\mathrm{CaC}_{6}$ results from the electron-phonon interaction is the observation of the $\mathrm{Ca}$ isotope effect [25]. In particular, the experiment has reported the high value of the isotope exponent, close to the BCS limit: $\alpha_{\mathrm{Ca}} \sim 0.5[3,4]$. This data suggests the dominant role of the $\mathrm{Ca}$ phonons in the electron-phonon coupling.

In the present study, we have analyzed the $\mathrm{CaC}_{6}$ superconducting state, at the ambient pressure, in order to determine the effect of anisotropy on the values of the thermodynamic functions. For comparison purposes, the results obtained in the one-band approach have been also presented.

\section{The formalism}

\subsection{The one-band Eliashberg equations}

In the simplest case, the properties of the superconducting state in $\mathrm{CaC}_{6}$ have been analyzed with the use of the oneband Eliashberg equations determined on the imaginary axis $(i \equiv \sqrt{-1})[5,26]$ :

$$
\Delta_{n} Z_{n}=\pi k_{B} T \sum_{m=-M}^{M} \frac{\left[K\left(\omega_{n}-\omega_{m}\right)-\mu^{\star}\left(\omega_{m}\right)\right]}{\sqrt{\omega_{m}^{2}+\Delta_{m}^{2}}} \Delta_{m}
$$

and

$$
Z_{n}=1+\pi k_{B} T \sum_{m=-M}^{M} \frac{K\left(\omega_{n}-\omega_{m}\right)}{\sqrt{\omega_{m}^{2}+\Delta_{m}^{2}}} \frac{\omega_{m}}{\omega_{n}} Z_{m}
$$

where the symbol $\Delta_{n} \equiv \Delta\left(i \omega_{n}\right)$ denotes the order parameter, and $Z_{n} \equiv Z\left(i \omega_{n}\right)$ is the wave function renormalization factor. The Matsubara frequency has been defined in the following way: $\omega_{n} \equiv \pi k_{B} T(2 n-1)$, whereas $k_{B}$ represents the Boltzmann constant.

The form of the electron-phonon interaction has been determined by the properties of the pairing kernel:

$$
K\left(\omega_{n}-\omega_{m}\right) \equiv 2 \int_{0}^{+\infty} \frac{\alpha^{2} F(\Omega) \Omega}{\left(\omega_{n}-\omega_{m}\right)^{2}+\Omega^{2}},
$$

where $\alpha^{2} F(\Omega)$ is the Eliashberg function.

The depairing electron correlations have been modeled in the considered formalism with the use of the function: $\mu^{\star}\left(\omega_{m}\right) \equiv \mu^{\star} \theta\left(\omega_{c}-\left|\omega_{m}\right|\right)$, where $\mu^{\star}$ represents the Coulomb pseudopotential; $\theta$ is the Heaviside's function, and $\omega_{c}$ denotes the cut-off frequency.

The one-band Eliashberg equations have been solved in the presented article for 3601 Matsubara frequencies $(M=$ 1800). The numerical methods tested in the works [27-31] have been used. The stable solutions have been obtained for $T \geq T_{0}=2 \mathrm{~K}$.

\subsection{The three-band Eliashberg equations}

The anisotropic properties of the superconducting state in $\mathrm{CaC}_{6}$ should be examined in the framework of the threeband Eliashberg formalism. The corresponding equations take the form:

$$
\begin{aligned}
& \Delta_{n}^{\alpha} Z_{n}^{\alpha}=\pi k_{B} T \\
& \times \sum_{\beta \in\{a, b, c\}} \sum_{m=-N}^{N} \frac{\left[K^{\alpha \beta}\left(\omega_{n}-\omega_{m}\right)-\mu_{\alpha \beta}^{\star}\left(\omega_{m}\right)\right]}{\sqrt{\omega_{m}^{2}+\left(\Delta_{m}^{\beta}\right)^{2}}} \Delta_{m}^{\beta}
\end{aligned}
$$

and

$$
\begin{aligned}
Z_{n}^{\alpha}= & 1+\pi k_{B} T \\
& \times \sum_{\beta \in\{a, b, c\}} \sum_{m=-N}^{N} \frac{K^{\alpha \beta}\left(\omega_{n}-\omega_{m}\right)}{\sqrt{\omega_{m}^{2}+\left(\Delta_{m}^{\beta}\right)^{2}}} \frac{\omega_{m}}{\omega_{n}} Z_{m}^{\beta},
\end{aligned}
$$

where $\alpha$ denotes the band index. In particular: $\alpha \in$ $\{a, b, c\}$. From the physical point of view, the effective bands in $\mathrm{CaC}_{6}$ have been distinguished due to the breakdown of the Fermi surface into three separate parts, namely: (a) the external $\pi$ surface; (b) the Ca sphere; and (c) the internal $\pi$ surface [17].

In the considered case, the pairing kernels $\left(K^{\alpha \beta}\left(\omega_{n}-\omega_{m}\right)\right)$ can be calculated with the help of the formula below:

$$
K^{\alpha \beta}\left(\omega_{n}-\omega_{m}\right)=\lambda^{\alpha \beta} \frac{\Omega_{C}^{2}}{\left(\omega_{n}-\omega_{m}\right)^{2}+\Omega_{C}^{2}},
$$

where the symbols $\lambda^{\alpha \beta}$ denote the coupling constants.

The quantity $\Omega_{C}$ represents the characteristic phonon frequency, which value should be calculated numerically.

The depairing electron correlations have been determined by the functions: $\mu_{\alpha \beta}^{\star}\left(\omega_{m}\right) \equiv \mu_{\alpha \beta}^{\star} \theta\left(\omega_{c}-\left|\omega_{m}\right|\right)$, 

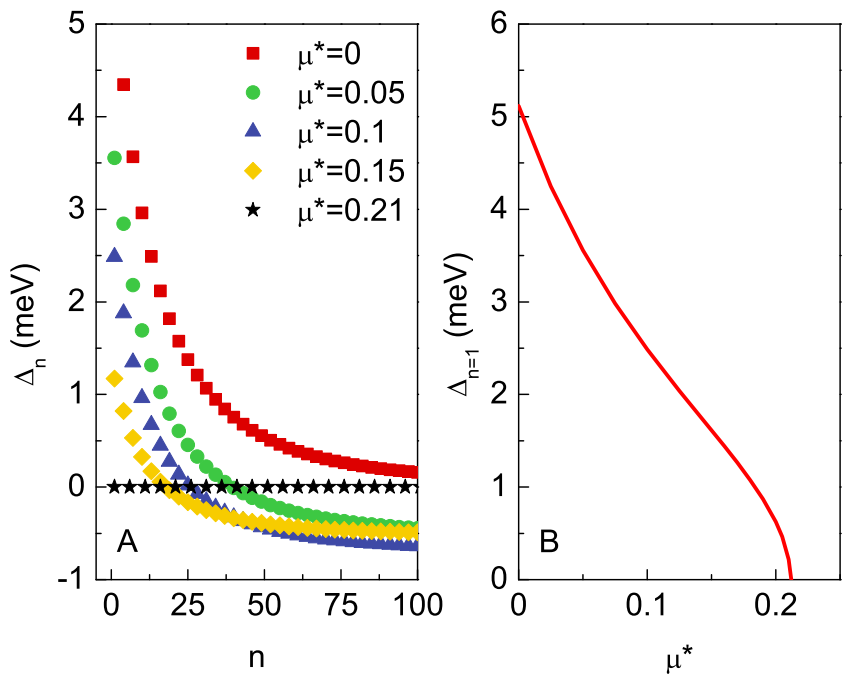

Fig. 1. The one-band formalism: (A) the form of the order parameter on the imaginary axis for the selected values of the Coulomb pseudopotential $\left(T=T_{C}\right)$. The results for the first 100 Matsubara frequencies have been presented. (B) The dependence of the maximum value of the order parameter on the Coulomb pseudopotential.

where the parameters $\mu_{\alpha \beta}^{\star}$ denote the anisotropic Coulomb pseudopotentials.

The three-band Eliashberg equations for $\mathrm{CaC}_{6}$ have been solved for 1201 Matsubara frequencies $(N=600)$. The numerical packages tested in the works $[11,15,32,33]$ have been used. The stable solutions have been obtained for $T \geq T_{0}=2 \mathrm{~K}$.

\section{The input parameters}

The Eliashberg equations have two input parameters: the Eliashberg function and the Coulomb pseudopotential. Eventually, the Eliashberg function can be replaced by the respective coupling constant and the characteristic phonon frequency.

In the one-band case, for the description of the properties of the superconducting state in $\mathrm{CaC}_{6}$, the Eliashberg function calculated in the work [34] has been used. The corresponding value of the coupling constant $(\lambda)$ is equal to 0.831 . The maximum value of the phonon frequency $\left(\Omega_{\max }\right)$ equals $183.4 \mathrm{meV}$. Additionally, $\omega_{c}=10 \Omega_{\max }$ has been assumed.

The value of the Coulomb pseudopotential has been calculated with the help of the equation: $\left[\Delta_{n=1}\left(\mu^{\star}\right)\right]_{T=T_{C}}=0$. It is worth noting that the form of the order parameter on the imaginary axis for the selected values of $\mu^{\star}$ is presented in Figure 1A. Additionally, Figure $1 \mathrm{~B}$ presents the full course of the function $\Delta_{n=1}\left(\mu^{\star}\right)$. On the basis of the presented results, it has been found that the value of the parameter $\mu^{\star}$ equals 0.21 . From the physical point of view, it is a relatively high value, which means that the depairing electron correlations are significant in $\mathrm{CaC}_{6}$ compound.

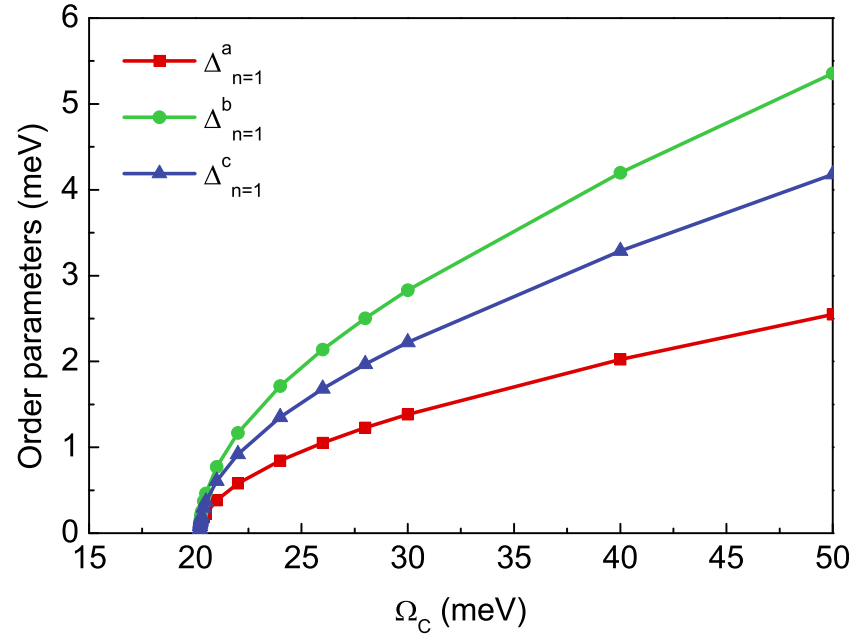

Fig. 2. The three-band formalism: the dependence of the order parameters on the value of the characteristic phonon frequency $\left(T=T_{C}\right)$.

In the three-band case, the matrix of the electronphonon coupling constants has been determined numerically in the work [17]. In particular, it has been obtained:

$$
\left[\lambda^{\alpha \beta}\right]=\left[\begin{array}{lll}
0.286 & 0.173 & 0.223 \\
0.518 & 0.315 & 0.425 \\
0.382 & 0.245 & 0.303
\end{array}\right] .
$$

The matrix of the Coulomb pseudopotentials has been calculated in the next step:

$$
\begin{aligned}
{\left[\mu_{\alpha \beta}^{\star}\right] } & =\mu_{3}^{\star}\left[\begin{array}{lll}
\frac{\rho_{a}(0)}{\rho(0)} & \frac{\rho_{a}(0)}{\rho(0)} & \frac{\rho_{a}(0)}{\rho(0)} \\
\frac{\rho_{b}(0)}{\rho(0)} & \frac{\rho_{b}(0)}{\rho(0)} & \frac{\rho_{b}(0)}{\rho(0)} \\
\frac{\rho_{c}(0)}{\rho(0)} & \frac{\rho_{c}(0)}{\rho(0)} & \frac{\rho_{c}(0)}{\rho(0)}
\end{array}\right] \\
& =\left[\begin{array}{lll}
0.108 & 0.108 & 0.108 \\
0.038 & 0.038 & 0.038 \\
0.063 & 0.063 & 0.063
\end{array}\right] .
\end{aligned}
$$

Let us note that the parameter $\mu_{3}^{\star}$ is equal to 0.21 [35], whereas the electron density of states vector has the following form [17]:

$$
\left[\rho_{\alpha}(0)\right]=\left[\begin{array}{c}
0.79 \\
0.27 \\
0.45
\end{array}\right]\left[\frac{\text { states }}{\mathrm{eV} * \text { cell }}\right],
$$

where $\rho(0)=\sum_{\alpha} \rho_{\alpha}(0)$.

In the last step, having the overt form of the matrix $\left[\lambda^{\alpha \beta}\right]$ and $\left[\mu_{\alpha \beta}^{\star}\right]$ at the disposal, the value of the characteristic phonon frequency has been determined. Up to this point, the equation: $\left[\Delta_{n=1}^{\alpha}\left(\Omega_{C}\right)\right]_{T=T_{C}}=0$ has been solved. As a result, it has been obtained: $\Omega_{C}=20.21 \mathrm{meV}$. The full dependence of the parameters $\Delta_{n=1}^{\alpha}$ on $\Omega_{C}$ has been presented in Figure 2.

Next, the relationship between the input parameters for the one-band and the three-band case has been discussed. Let us recall that in the one-band formalism, it has 

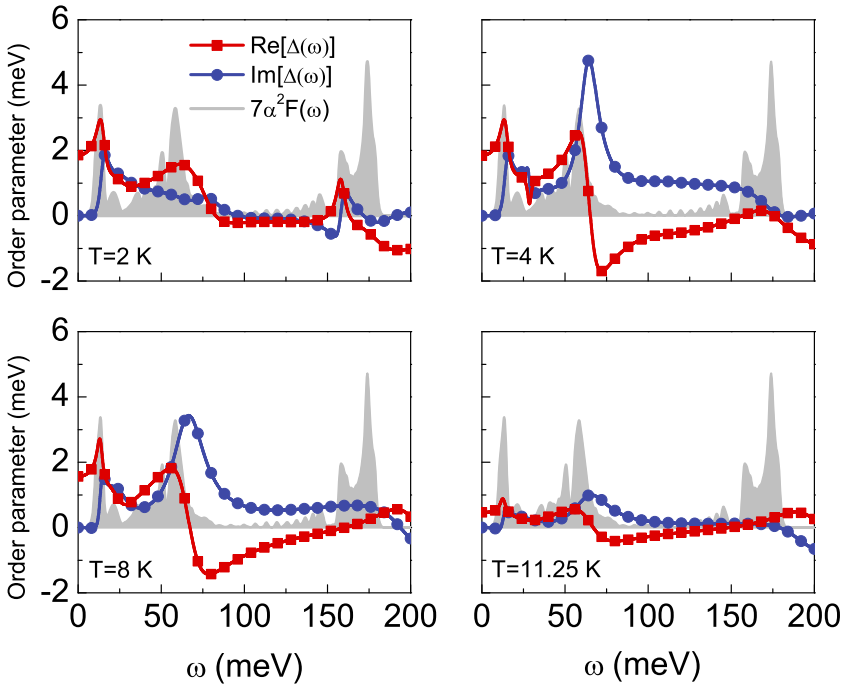

Fig. 3. The one-band formalism: the form of the order parameter on the real axis for the selected values of the temperature. The rescaled Eliashberg function is additionally presented in the figure.

been obtained: $\lambda=0.831$ and $\mu^{\star}=0.21$. Of course, in the three-band formalism we do not have scalar input parameters but matrices: $\left[\lambda^{\alpha \beta}\right]$ and $\left[\mu_{\alpha \beta}^{\star}\right]$. Note however, that with their help, we can determine the values corresponding to the values $\lambda$ and $\mu^{\star}$. In particular, according to the method proposed by Suhl et al., all the eigenvalues of the matrices must be calculated and their highest values must be taken into account [36]. In the present case, it has been obtained: $\langle\lambda\rangle=0.911$ and $\left\langle\mu^{\star}\right\rangle=0.21$. Based on the above results, it can be seen that the parameters $\langle\lambda\rangle$ and $\left\langle\mu^{\star}\right\rangle$ agree with the results obtained in the framework of the one-band formalism.

\section{The order parameter}

The exact value of the order parameter can be obtained by the analytic continuation of the $\Delta_{m}$ function on the real axis $(\omega)[37]$.

The courses of the order parameter on the real axis, obtained in the framework of the one-band formalism, are presented in Figure 3. The selected values of the temperature have been taken into account. It can be noticed that for lower frequencies the non-zero is only the real part of the function $\Delta(\omega)$. The strong damping effects are induced for the higher frequencies in $\mathrm{CaC}_{6}$ compound. Those effects are described by the imaginary part of the order parameter [38]. Additionally, the rescaled Eliashberg function has been plotted in Figure 3. It can be clearly observed that the functions $\operatorname{Re}[\Delta(\omega)]$ and $\operatorname{Im}[\Delta(\omega)]$ are correlated with the shape of the spectral function. For this reason, the values of the order parameter are worth presenting also on the complex plane. The obtained results have been collected in Figure 4. It has been found that the values of the order parameter create the characteristic

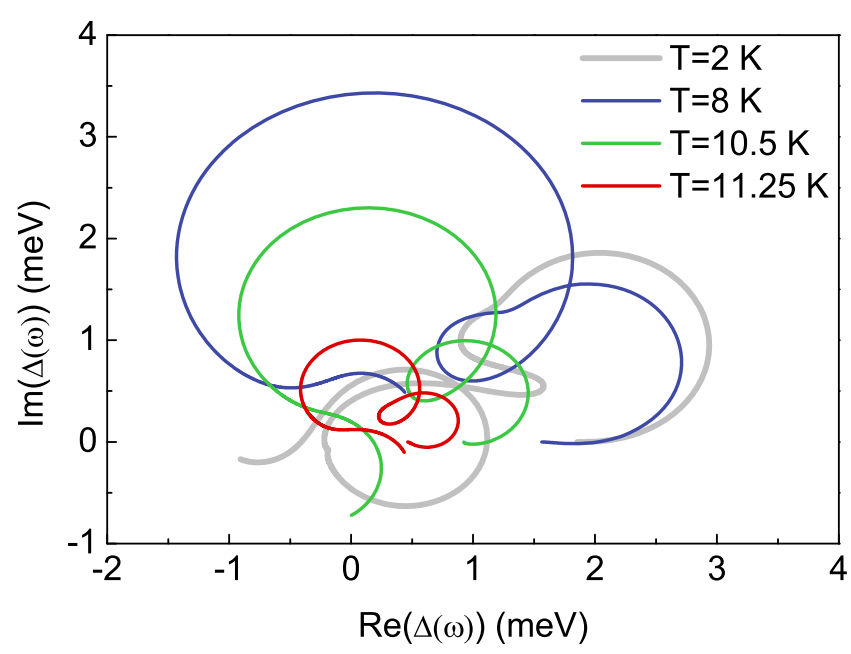

Fig. 4. The one-band formalism: the form of the order parameter on the complex plane for the selected values of the temperature.

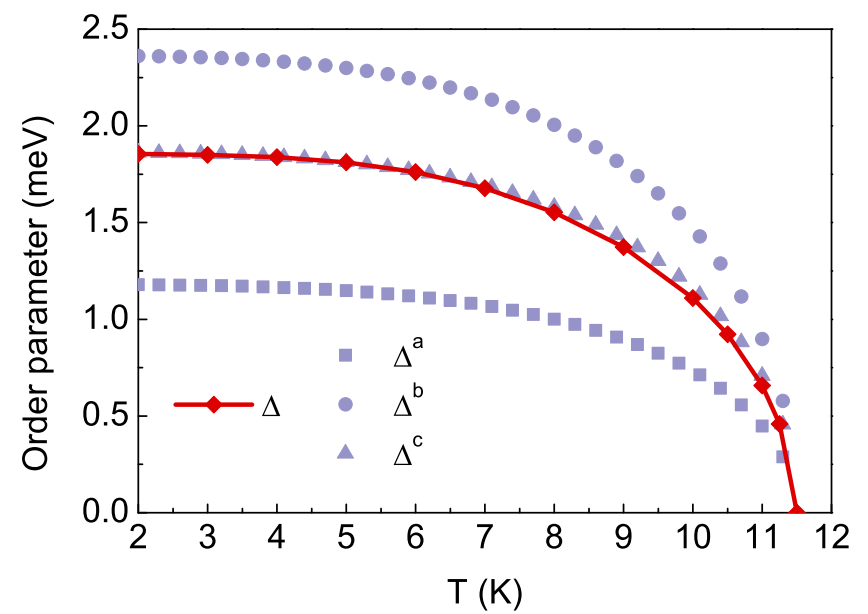

Fig. 5. The influence of the temperature on the physical value of the order parameter. The red line with squares has been obtained in the framework of the one-band model. The remaining symbols present the results of the three-band approach.

deformed spirals with the radii decreasing together with the increasing temperature.

It should be noted that much smoother functions of the order parameter than in the one-band case appear in the three-band case, which results from the replacement of the spectral functions in the Eliashberg equations by the coupling constants and the characteristic phonon frequency.

The physical value of the order parameter for the given temperature has been calculated on the basis of the following formula:

$$
\Delta(T)=\operatorname{Re}[\Delta(\omega=\Delta(T), T)] .
$$

The obtained results have been plotted in Figure 5 . It has been found that strong anisotropy of the order parameter, visible substantially throughout the entire considered temperature range, appears in the superconducting state in $\mathrm{CaC}_{6}$ compound. 
Let us note that the values of the order parameter presented in Figure 5 can be calculated with the help of the simple formula:

$$
\Delta^{\alpha}(T)=\Delta^{\alpha}(0) \sqrt{1-\left(\frac{T}{T_{C}}\right)^{\Gamma}},
$$

wherein for the one-band case, it has been obtained: $\Delta(0) \equiv \Delta\left(T_{0}\right)=1.855 \mathrm{meV}$.

The multiband formalism gives the following results: $\Delta^{a}(0)=1.180 \mathrm{meV}, \Delta^{b}(0)=2.364 \mathrm{meV}, \Delta^{c}(0)=$ $1.864 \mathrm{meV}$. In both cases $\Gamma=7 / 2$. Please note that in the framework of the BCS formalism, the parameter $\Gamma$ is equal to 3 [39].

Based on the presented results, the values of the dimensionless ratios have been estimated: $R_{\Delta}^{\alpha} \equiv$ $2 \Delta^{\alpha}(0) / k_{B} T_{C}$.

Let us note that the value of the parameter $R_{\Delta}$ is the universal constant of the BCS model and it is equal to $3.53[3,4]$. In the one-band case, it has been obtained: $R_{\Delta}=3.77$, whereas the anisotropic description gives: $R_{\Delta}^{a}=2.40, R_{\Delta}^{b}=4.83$, and $R_{\Delta}^{c}=3.80$.

When comparing the result obtained in the framework of the one-band formalism with the value predicted by the BCS theory, it is clear that the parameter $R_{\Delta}$ is slightly higher than $\left[R_{\Delta}\right]_{\mathrm{BCS}}$. This result means that the strongcoupling and the retardation effects in $\mathrm{CaC}_{6}$ compound do not significantly affect the value of $R_{\Delta}$.

The considered effects can be characterized quantitatively in the framework of the Eliashberg formalism by calculating the ratio: $r \equiv k_{B} T_{C} / \omega_{\ln }$, where $\omega_{\ln }$ is the logarithmic phonon frequency. In the case of $\mathrm{CaC}_{6}$, we have: $\omega_{\mathrm{ln}}=27.78 \mathrm{meV}$. Thus, the parameter $r$ equals 0.036 . On the other hand, in the BCS limit, the Eliashberg equations predict: $r=0[6]$.

However, the situation is subject to a significant change, when we take into account the anisotropy of the studied system. Based on the obtained results, it can be easily noticed that $R_{\Delta}$ in the bands $a$ and $b$ significantly deviates from $\left[R_{\Delta}\right]_{\mathrm{BCS}}$.

With the explicit forms of the order parameter functions we can, in a relatively simple way, plot a graph of the total normalized density of states:

$$
\frac{N_{S}(\omega)}{N_{N}(\omega)} \equiv \sum_{\alpha \in\{a, b, c\}} \chi_{\alpha} \frac{N_{S}^{\alpha}(\omega)}{N_{N}^{\alpha}(\omega)},
$$

where $N_{S}^{\alpha}(\omega)$ and $N_{N}^{\alpha}(\omega)$ denote the density functions for the superconducting state and the normal state, respectively. Additionally, for the anisotropic case it has been assumed that: $\chi_{\alpha}=1 / 3$.

The normalized band densities of states have been calculated on the basis of the formula below:

$$
\frac{N_{S}^{\alpha}(\omega)}{N_{N}^{\alpha}(\omega)}=\operatorname{Re}\left[\frac{|\omega-i \delta|}{\sqrt{(\omega-i \delta)^{2}-\left(\Delta^{\alpha}(\omega)\right)^{2}}}\right],
$$

whereas the pair breaking parameter $\delta$ is equal to $0.1 \mathrm{meV}$.

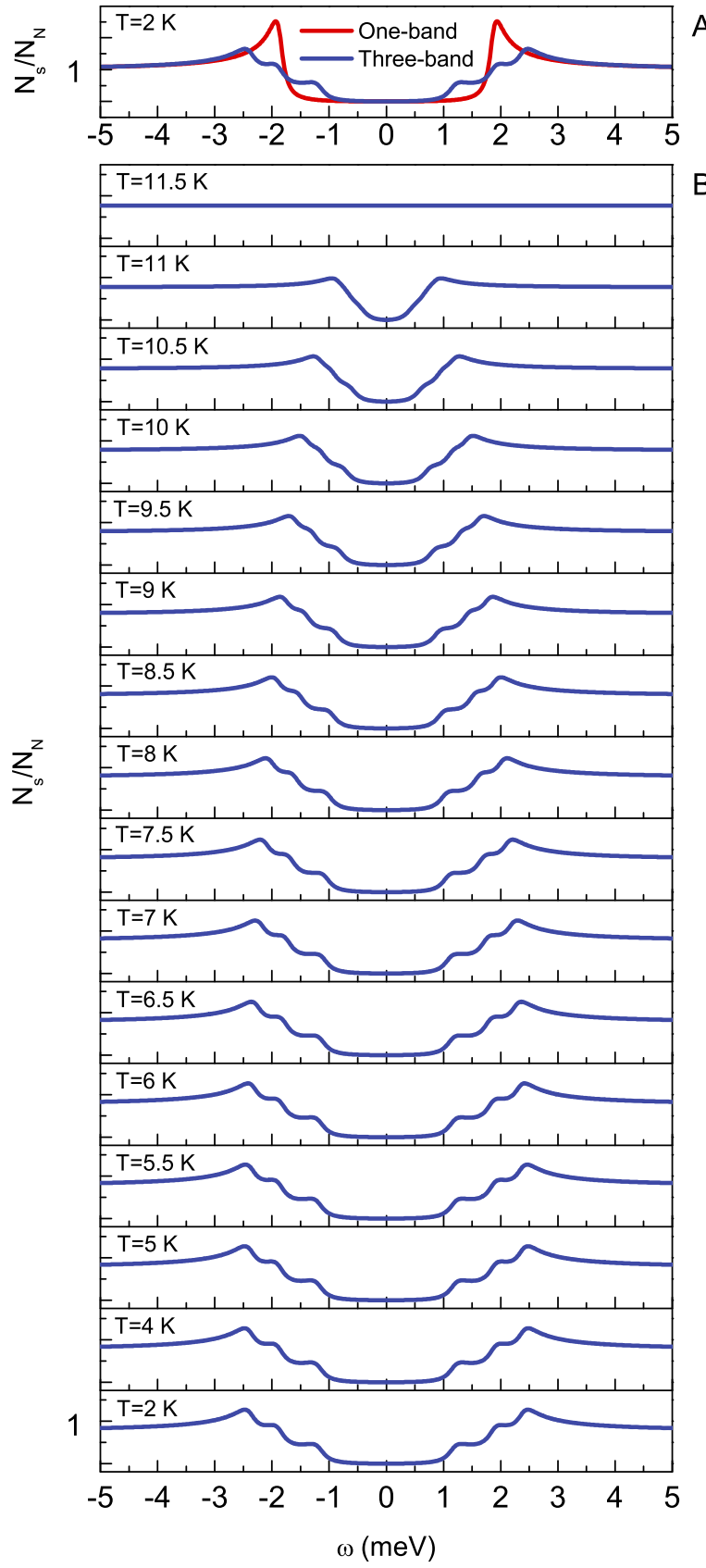

Fig. 6. The one-band and the three-band formalism: the total normalized density of states. (A) The comparison of the oneband results and the three-band results for the lowest considered temperature. (B) The dependence of the total normalized density of states on the temperature in the three-band case.

The total normalized density of states calculated in the framework of the one- and three-band model has been plotted in Figure 6. On the basis of the presented data, it has been found that in the case of $\mathrm{CaC}_{6}$, the anisotropy of the superconducting state causes a clear split of two maxima of the single-band function into six maxima in the three-band case. It is worth noting that the maxima are situated at the points of incidence with the value of the frequency equal to $\pm \Delta^{\alpha}$. 


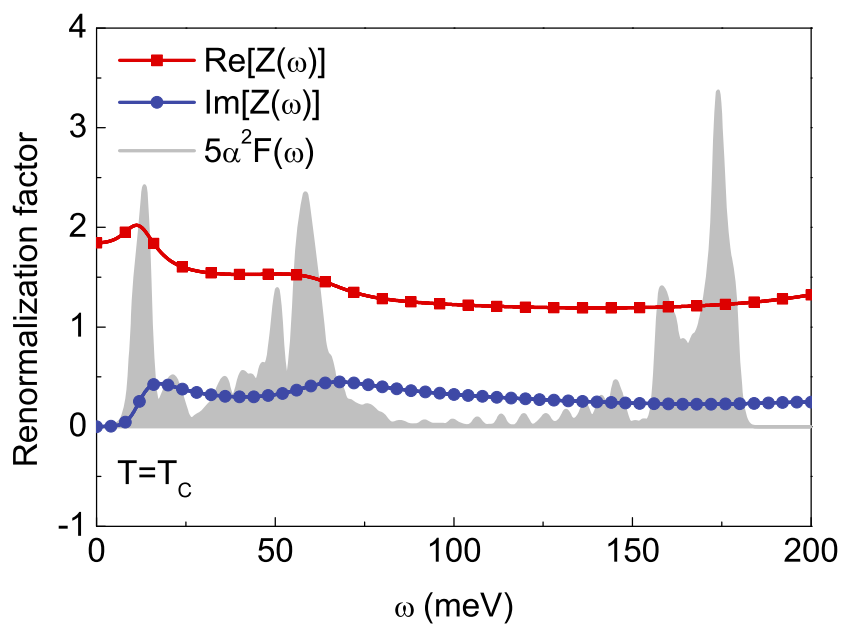

Fig. 7. The one-band formalism: the wave function renormalization factor on the real axis at the critical temperature. Additionally, the figure presents the rescaled Eliashberg function.

\section{The wave function renormalization factor}

Next, we have analyzed the influence of anisotropy on the form of the second solution of the Eliashberg equations.

The physical value of the renormalization factor can be obtained by the analytic continuation of the $Z_{n}$ function on the real axis [37].

When analyzing the obtained results, it has been found that both in the one-band and in the three-band case, the renormalization factor very slightly depends on the temperature. Based on the above statement, Figure 7 presents only the form of the function $Z(\omega)$ at the critical temperature. Additionally, a graph of the rescaled Eliashberg function is presented in the figure. It has been found that, like as in the case of the order parameter, the non-zero values for lower frequencies are taken only by the real part of the function $Z(\omega)$. For higher frequencies both $\operatorname{Re}[Z(\omega)]$ and $\operatorname{Im}[Z(\omega)]$ are characterized by the complicated forms, wherein the shapes of the considered functions are clearly correlated with the shape of the Eliashberg function.

The electron effective mass $\left(m_{e}^{\star}\right)^{\alpha}$ should be calculated in the framework of the Eliashberg theory with the help of the following formula:

$$
\left(m_{e}^{\star}\right)^{\alpha}=\operatorname{Re}\left[Z^{\alpha}(\omega=0, T)\right]\left(m_{e}\right)^{\alpha},
$$

where the symbol $\left(m_{e}\right)^{\alpha}$ denotes the electron band mass.

A full dependence of the electron effective mass on temperature for the one-band and the three-band case is presented in Figure 8. On the basis of the presented data, it has been found that the anisotropy of the electron effective mass is clearly visible in the entire range of the existence of the superconducting state. It is also worth noting that the highest effective mass belongs to the electrons of the $b$ band, which is directly related to the high value of the effective coupling constant for the considered band $\left(\lambda^{b} \equiv \sum_{\beta \in\{a, b, c\}} \lambda^{b \beta}=1.258\right)$.

In the last step let us note that the influence of temperature on the value of the electron effective mass

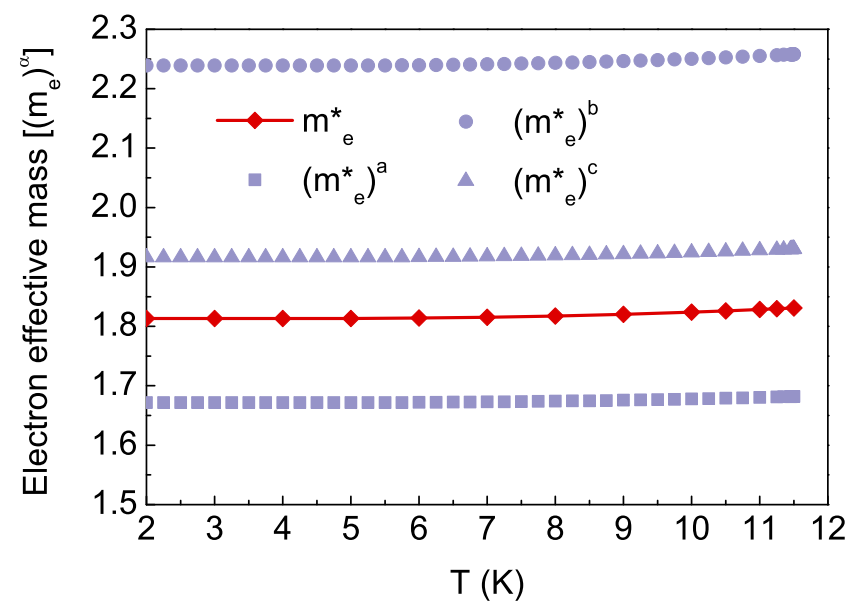

Fig. 8. The dependence of the electron effective mass on the temperature. The solid line has been obtained in the framework of the one-band model. The symbols represent the results of the three-band approach.

can be characterized analytically. For this purpose, we have introduced the auxiliary indication: $Z^{\alpha}(T) \equiv$ $\operatorname{Re}\left[Z^{\alpha}(\omega=0, T)\right]$. It has turned out that the function $Z^{\alpha}(T)$ can be parameterized using the following formula:

$$
Z^{\alpha}(T)=Z^{\alpha}(0)+\left[Z^{\alpha}\left(T_{C}\right)-Z^{\alpha}(0)\right]\left(\frac{T}{T_{C}}\right)^{\Gamma},
$$

where: $Z^{\alpha}(0)=Z^{\alpha}\left(T_{0}\right)$ and $Z^{\alpha}\left(T_{C}\right) \simeq 1+$ $\sum_{\beta \in\{a, b, c\}} \lambda^{\alpha \beta}$. In the one-band case, it has been obtained: $Z(0)=1.813$ and $Z\left(T_{C}\right)=1.831$, whereas the three-band formalism predicts:

$Z_{n=1}^{a}(0)=1.672, \quad Z_{n=1}^{b}(0)=2.239, \quad Z_{n=1}^{c}(0)=1.916$,

$Z_{n=1}^{a}\left(T_{C}\right)=1.682, \quad Z_{n=1}^{b}\left(T_{C}\right)=2.258$,

and $Z_{n=1}^{c}\left(T_{C}\right)=1.930$.

\section{The thermodynamic critical field and the specific heat jump}

In order to determine the thermodynamic critical field and the specific heat jump, it is necessary to calculate the free energy difference between the superconducting and the normal state [40]:

$$
\begin{aligned}
\Delta F= & -2 \pi k_{B} T \sum_{m=1}^{M} \sum_{\alpha \in\{a, b, c\}} \rho_{\alpha}(0) \\
& \times\left[\sqrt{\omega_{m}^{2}+\left(\Delta_{m}^{\alpha}\right)^{2}}-\left|\omega_{m}\right|\right] \\
& \times\left[Z_{m}^{\alpha,(S)}-Z_{m}^{\alpha,(N)} \frac{\left|\omega_{m}\right|}{\sqrt{\omega_{m}^{2}+\left(\Delta_{m}^{\alpha}\right)^{2}}}\right],
\end{aligned}
$$




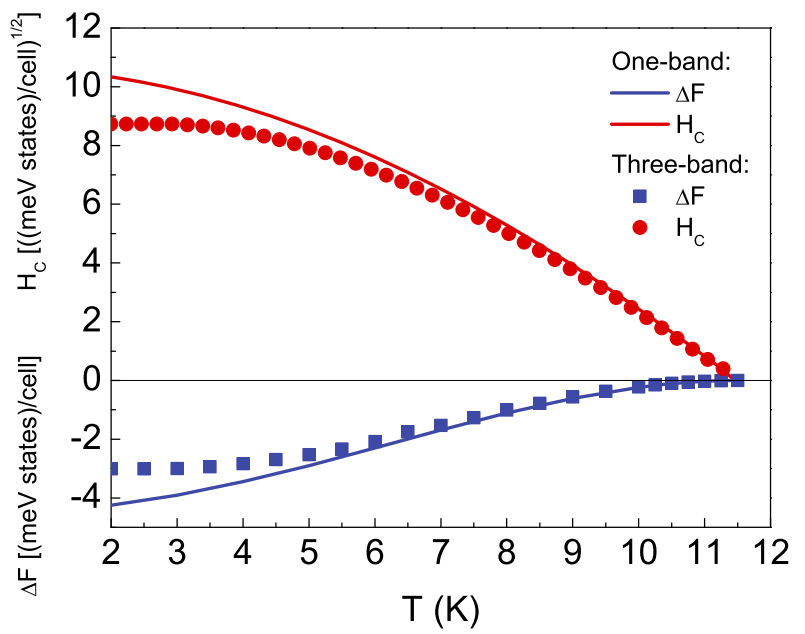

Fig. 9. The dependence of the free energy and the thermodynamic critical field on the temperature. The solid line has been obtained in the framework of the one-band model. The symbols represent the three-band approach.

where the upper indexes $S$ and $N$ denote the superconducting and the normal state, respectively. In the oneband case, the electron density of states on the Fermi surface is equal to: $\rho(0)=1.5$ states $/ \mathrm{eV}^{*}$ cell [41].

The results obtained for the free energy difference (the one-band and the three-band case) are presented in the lower panel of Figure 9. It can be noticed that anisotropy causes a clear increase in the values of $\Delta F$ in the range of low temperatures. In the case of the temperatures close to the critical temperature, the isotropic and the anisotropic results are virtually indistinguishable.

The values of the thermodynamic critical field have been calculated on the basis of the following formula:

$$
H_{C}=\sqrt{-8 \pi \Delta F} .
$$

The numerical results are presented in the upper panel of Figure 9. Due to the form of the free energy difference, the influence of anisotropy on the values of $H_{C}$ is clearly visible only in the range of low temperatures.

In the last step, the specific heat jump at the critical temperature has been calculated: $\left(\Delta C\left(T_{C}\right) \equiv C^{(S)}\left(T_{C}\right)-\right.$ $\left.C^{(N)}\left(T_{C}\right)\right)$. Due to the fact that the function $\Delta F$ has an identical form near the critical temperature, for the oneand three-band case the following formula has been used:

$$
\Delta C=-T \frac{d^{2} \Delta F}{d T^{2}}
$$

The obtained result can be interpreted in the most convenient way by calculating the value of the dimensionless ratio: $R_{C} \equiv \Delta C\left(T_{C}\right) / C^{(N)}\left(T_{C}\right)$, where the specific heat of the normal state is given by the formula: $\frac{C^{N}(T)}{k_{B} \rho(0)}=\frac{\gamma}{\beta}$. The Sommerfeld constant is given by: $\gamma \equiv \frac{2}{3} \pi^{2}(1+\lambda)$. For $\mathrm{CaC}_{6}$ compound, it has been obtained: $R_{C}=1.71$. Let us notice that the classical BCS theory predicts: $R_{C}=$ $1.43[3,4]$. This result means that the strong-coupling and the retardation effects in $\mathrm{CaC}_{6}$ compound increase the value of the parameter $R_{C}$ in comparison to the result predicted by the BCS model.

\section{Summary}

The presented work describes the influence of anisotropy of the electron-phonon and the electron-electron interaction on the values of the thermodynamic functions of the superconducting state in $\mathrm{CaC}_{6}$ compound.

The numerical calculations have been carried out in the framework of the isotropic and the anisotropic (threeband) Eliashberg formalism.

It has been found that anisotropy significantly changes the values of the order parameter. In particular, the isotropic value of the order parameter for $T=T_{0}$ is equal to $1.855 \mathrm{meV}$. In the anisotropic case, the minimum and the maximum value of the order parameter equals: $1.180 \mathrm{meV}$ and $2.364 \mathrm{meV}$, respectively. Note that this result implies a significant change in the form of the total normalized density of states in $\mathrm{CaC}_{6}$ compound when compared to the predictions of the isotropic formalism.

The anisotropy of the pairing and the depairing interaction has additionally a significant effect on the value of the electron effective mass. In the isotropic case, for $T=T_{C}$ it has been obtained: $m_{e}^{\star}=1.831 m_{e}$, whereas the anisotropic calculations determine the extension of the effective mass from $1.682\left(m_{e}\right)^{a}$ to $2.258\left(m_{e}\right)^{b}$.

It has been also shown that the anisotropy of the interactions in $\mathrm{CaC}_{6}$ visibly lowers the low-temperature values of the thermodynamic critical field. On the other hand, it has no effect on the specific heat jump at the critical temperature.

We note also that presented here results can be of fundamental and practical interest not only in the terms of the bulk carbon intercalated superconductors, but also in the discussion on the possible superconductivity in the low-dimensional graphene-based materials $[42,43]$, following recently published work by Yang et al. [44].

Finally, we would like to underline, that the thermodynamic properties calculated in the present work should change under the pressure, corresponding to the relation between the critical temperature and pressure, as described in the introduction. This vague and interesting problem is currently under our investigation.

In memory of Marian Szczęśniak, on the first anniversary of his death. The Authors are thankful to K. Dziliński and Z. Bạk for their kindness and support throughout this work. Some calculations have been conducted on the Częstochowa University of Technology cluster, built in the framework of the PLATON project No. POIG.02.03.00-00-028/08 - the service of the campus calculations U3.

\section{References}

1. H. Fröhlich, Phys. Rev. 79, 845 (1950)

2. H. Fröhlich, Proc. R. Soc. A 223, 296 (1954)

3. J. Bardeen, L.N. Cooper, J.R. Schrieffer, Phys. Rev. 106, $162(1957)$ 
4. J. Bardeen, L.N. Cooper, J.R. Schrieffer, Phys. Rev. 108, 1175 (1957)

5. G.M. Eliashberg, Soviet. Phys. J. Exp. Theor. Phys. 11, $696(1960)$

6. J.P. Carbotte, F. Marsiglio, in The Physics of Superconductors, edited by K.H. Bennemann, J.B. Ketterson (Springer, Berlin, 2003), Vol 1, p. 223

7. A.L. Fetter, J.D. Walecka, Quantum Theory of Manyparticle Systems (McGraw-Hill Book Company, 1971)

8. K. Elk, W. Gasser, Die Methode der Greenschen Funktionen in der Festkörperphysik (Akademie-Verlag, Berlin, 1979)

9. J. Nagamatsu, N. Nakagawa, T. Muranaka, Y. Zenitani, J. Akimitsu, Nature 410, 63 (2001)

10. A.A. Golubov, J. Kortus, O.V. Doglov, O. Jepsen, Y. Kong, O.K. Andersen, B.J. Gibson, K. Ahn, R.K. Kremer, J. Phys.: Condens. Matter 14, 1353 (2002)

11. R. Szczȩśniak, Solid State Commun. 145, 137 (2008)

12. P. Cudazzo, G. Profeta, A. Sanna, A. Floris, A. Continenza, S. Massidda, E.K.U. Gross, Phys. Rev. Lett. 100, 257001 (2008)

13. P. Cudazzo, G. Profeta, A. Sanna, A. Floris, A. Continenza, S. Massidda, E.K.U. Gross, Phys. Rev. B 81, 134505 (2010)

14. P. Cudazzo, G. Profeta, A. Sanna, A. Floris, A. Continenza, S. Massidda, E.K.U. Gross, Phys. Rev. B 81, 134506 (2010)

15. R. Szczȩśniak, E.A. Drzazga, Solid State Sci. 19, 167 (2013)

16. N. Emery, C. Herold, M. d'Astuto, V. Garcia, Ch. Bellin, J.F. Mareche, P. Lagrange, G. Loupias, Phys. Rev. Lett. 95, 087003 (2005)

17. S. Massidda, F. Bernardini, C. Bersier, A. Continenza, P. Cudazzo, A. Floris, H. Glawe, M. Monni, S. Pittalis, G. Profeta, A. Sanna, S. Sharma, E.K.U. Gross, Supercond. Sci. Technol. 22, 034006 (2009)

18. J.S. Kim, L. Boeri, R.K. Kremer, F.S. Razavi, Phys. Rev. B 74, 214513 (2006)

19. G. Lamura, M. Aurino, G. Cifariello, E. Di Gennaro, A. Andreone, N. Emery, C. Herold, J.-F. Mareche, P. Lagrange, Phys. Rev. Lett. 96, 107008 (2006)

20. R.P. Smith, A. Kusmartseva, Y.T.C. Ko, S.S. Saxena, A. Akrap, L. Forró, M. Laad, T.E. Weller, M. Ellerby, N.T. Skipper, Phys. Rev. B 74, 024505 (2006)

21. L. Zhang, Y. Xie, T. Cui, Y. Li, Z. He, Y. Ma, G. Zou, Phys. Rev. B 74, 184519 (2006)

22. A. Gauzzi, S. Takashima, N. Takeshita, C. Terakura, H Takagi, N. Emery, C. Herold, L. Lagrange, G. Loupias, Phys. Rev. Lett. 98, 067002 (2007)
23. M. Debessai, J.J. Hamlin, J.S. Schilling, D. Rosenmann, D.G. Hinks, H. Claus, Phys. Rev. B 82, 132502 (2010)

24. Y.-L. Li, W. Luo, X.-J. Chen, Z. Zeng, H.-Q. Lin, R. Ahuja, Sci. Rep. 3, 3331 (2013)

25. D.G. Hinks, D. Rosenmann, H. Claus, M.S. Bailey, J.D. Jorgensen, Phys. Rev. B 75, 014509 (2007)

26. J.P. Carbotte, Rev. Mod. Phys. 62, 1027 (1990)

27. R. Szczesśniak, D. Szczesśniak, E.A. Drzazga, Solid State Commun. 152, 2023 (2012)

28. R. Szczȩśniak, E.A. Drzazga, A.M. Duda, Solid State Commun. 166, 50 (2013)

29. R. Szczȩśniak, A.P. Durajski, J. Supercond. Nov. Magn. 25, 399 (2012)

30. R. Szczȩśniak, M. Mierzejewski, J. Zieliński, Physica C 355, 126 (2001)

31. R. Szczęśniak, D. Szczesśniak, Phys. Stat. Sol. B 249, 2194 (2012)

32. R. Szczȩśniak, Acta Physica Polonica A 109, 179 (2006)

33. R. Szczȩśniak, Solid State Commun. 138, 347 (2006)

34. M. Calandra, G. Profeta, F. Mauri, Phys. Rev. B 82, $165111(2010)$

35. A. Sanna, S. Pittalis, J.K. Dewhurst, M. Monni, S. Sharma, G. Ummarino, S. Massidda, E.K.U. Gross, arXiv: 1108.2800v2 (2012)

36. H. Suhl, B.T. Matthias, L.R. Walker, Phys. Rev. Lett. 3, $552(1959)$

37. K.S.D. Beach, R.J. Gooding, F. Marsiglio, Phys. Rev. B 61, 5147 (2000)

38. G. Varelogiannis, Z. Phys. B 104, 411 (1997)

39. H. Eschrig, Theory of Superconductivity - A Primer, private materials (2008)

40. J. Bardeen, M. Stephen, Phys. Rev. 136, A1485 (1964)

41. M. Calandra, F. Mauri, Phys. Rev. Lett. 95, 237002 (2005)

42. G. Profeta, M. Calandra, F. Mauri, Nat. Phys. 8, 131 (2012)

43. D. Szczȩśniak, A.P. Durajski, R. Szczȩśniak, J. Phys.: Condens. Matter 26, 255701 (2014)

44. S.-L. Yang, J.A. Sobota, C.A. Howard, C.J. Pickard, M. Hashimoto, D.H. Lu, S.-K. Mo, P.S. Kirchmann, Z.-X. Shen, Nat. Commun. 5, 3493 (2014)

Open Access This is an open access article distributed under the terms of the Creative Commons Attribution License (http://creativecommons.org/licenses/by/4.0), which permits unrestricted use, distribution, and reproduction in any medium, provided the original work is properly cited. 